\title{
Price Based Routing for Event Driven Prioritized Traffic in Wireless Sensor Networks
}

\author{
S. Yousaf Shah and Boleslaw K. Szymanski \\ Department of Computer Science and Network Science and Technology Center (NeST) \\ Rensselaer Polytechnic Institute (RPI), Troy, NY, USA \\ Email: \{shahs9, szymab\}@rpi.edu
}

\begin{abstract}
We present a dynamic price based routing protocol in which packets from different applications dynamically choose their paths by evaluating the price to be paid for taking each path and their ability to pay. We propose a mechanism in which the prices reflect congestion on routers and thus the waiting time for packet to pass through the router. These prices increase as usage of the usually preferred shorter routes increases. The packet's ability to pay price on a router is defined by the product of application's priority and the delay experienced at the router. As a result, the low priority applications intelligently avoid paths with high prices and go via low price routes. The low price routes may possibly be longer but require shorter waiting for passage at congested routers making them faster for low priority packets. This enables high priority traffic to get through quickly via shorter paths as they are able to pay high prices after little wait. Thus, our approach distributes traffic flows of different applications in the network and lowers congestion and delays for all applications. We further show that our dynamic path allocation technique ensures robust communication in fully functional as well as partially damaged networks. Our dynamic pricing mechanism quickly adapts routing to the damaged network, increases utilization of the partial network to lower the impact on critical infrastructure and key resources. Moreover, our proposed mechanism is equally applicable to both communication networks and physical infrastructure networks. ${ }^{1}$
\end{abstract}

Index Terms-Wireless Sensor Networks (WSNs); Quality of Information (QoI); Auctions; Congestion.

\section{INTRODUCTION}

Wireless sensor networks consist of sensing nodes that collect data from the environment and send it to one or more destinations. The data generated by sensing nodes increase significantly in volume when an event of interest happens in the monitored area. In event driven sensing, nodes start to generate huge volume of data often creating congestion on nodes that relay data to the destination. Various approaches have been proposed in the literature to route traffic from source to destination with minimized delay. Most of these approaches focus on congestion avoidance and congestion control techniques for continuous stream of data which increases the response time of these techniques to congestion. Although routing prioritized multi application traffic in sporadic event driven scenarios is relatively less explored area, recently there is growing interest in this area in the context of emergency services [1], [2]. In this paper, we explore mechanism that efficiently deals with sporadic traffic congestion by spreading traffic

\footnotetext{
${ }^{1}$ Initial unpublished manuscript is available on ACITA website which is an internal conference of ITA consortium.
}

with different priorities across the network. Networks laid to transport critical data, such as those providing connectivity to critical infrastructure and key resources (CIKR), require robustness against failures in emergency situations or events causing severe congestion. Here, we present a mechanism in which the packets intelligently choose time efficient paths for their route to the destination and can dynamically re-route in cases of partially damaged or unavailable networks.

Most of the ad-hoc routing algorithms attempt to route packets to destination via the shortest path, such as DSDV (Destination-Sequenced Distance Vector) or most efficient source route, such as Dynamic Source Routing (DSR) [3] etc. These protocols have the tendency to saturate such shortest paths and source routes. Especially in multi-priority applications, packets from every application follow the same shortest or efficient path to the destination, making it congested and increasing the delays. Under a fixed priority based route assignment scheme, the high priority packets always get through imposing either long delays or starvation for low priority applications in case of severe congestion. In the event driven scenarios, the events are intermittent and last for relatively short time, therefore typical rate control mechanisms or feedback loop approaches do not perform well. Furthermore, routing protocols that are designed for ad-hoc or on demand routing suffer from frequent path updates, which unnecessarily exhaust battery, and take long time to complete.

In multi application scenario, the data feeds from different applications have different priorities (often assigned by information consumer), therefore all applications cannot and should not be treated the same way. For example, data traffic originated or destined to CIKR should be of higher priority as compared to other traffic and therefore it should be delivered with lowest delay. The priority of an application is driven by several factors and may change over time depending upon the nature of the application and the network. Most often the priority reflects tolerance to delays; applications which are less tolerant to delays have higher priority.

Many multipath approaches dedicate routing paths to applications based on their priority. Such static allocation may lead to under utilization of the network because in many sensor network applications the traffic pattern constantly change. It is very difficult to predict the volume of data that will be flowing between any two nodes. There may be times when less data is produced for high priority applications while high 
data bursts are generated by the low priority applications. In such a case, the routes dedicated to high priority traffic are highly underutilized whereas routes dedicated to low priority applications get congested. Therefore, there is a need for a reactive routing mechanism that can respond to varying state of the network by dynamically adjusting routing paths for multi priority applications. Multipath routing is widely used technique for congestion resolution and increasing utilization of the network. Yet, as we discuss in the Related Work section, the proposed approaches have their limitations.

Congestion occurs not only in communication networks but also in the context of people movements such as during emergency building evacuation and/or on partially damaged travel routes. Evacuating people from a building in case of emergency without causing a stampede, efficiently routing traffic through heavily used toll booths, evacuating disaster stricken area and large exodus of population from a flood prone area to nearby safe towns all in essence are routing problems. Recently, researchers have started exploring sensor networks for improving building evacuations by designing intelligent building evacuation mechanisms [4], [5], [1] using sensor networks. In this paper, we also focus on the problem of distributed transportation of event driven traffic consisting of items (data packets, cars, people etc.) from one point to another under fully functional as well as partially damaged infrastructure.

We propose a routing protocol in which network traffic is routed through various dynamically chosen routes to destination. Routing nodes intelligently forward packets to nodes with lowest predicted delay. Whenever a router transmits a packet, auction among multiple possible paths is held. Path which incurs lowest delay for the application is selected and packet is forwarded to the immediate neighbor on such a path. The same process is repeated on the node which receives the packet. In such a way, every packet routing is based on its priority and the path price at the time. As the path prices change, the path chosen by the packets changes accordingly. A low price of a path may increase when high priority flow starts using the path and then low priority packets will dynamically change their paths to avoid long delays and diverge their flows from congested paths. Such dynamic divergence of traffic on distributed paths makes communication robust against damaged routes or routes experiencing huge delays which is of primary importance in networks connecting CIKR. Our mechanism not only keeps highly critical networks connected but also utilizes the surviving infrastructure efficiently to minimize delays.

Main contribution of this paper is the novel routing protocol that uses prices on the routing nodes and aging of the waiting packets to decide the next hop for each packet. The protocol has following features; (1) The protocol dynamically routes multi-priority traffic on different paths based on the auction winning prices on the routing nodes; (2) The protocol is resilient to network damages and adapts quickly in case network is partially damaged; (3) The protocol is also applicable to reducing delays in emergency evacuation scenarios over physical infrastructure networks.
The rest of the paper is organized as follows; in Section II, we show some motivating scenarios. Section III discusses related research. Section IV provides detail description of the mechanism and Section $\mathrm{V}$ shows performance results while Section VI concludes this paper.

\section{Motivating Scenarios}

In real-time monitoring, separating eventful and uneventful data is an important issue which usually does not get significant attention. The data feeds received from different sensors are of different importance. Congestion control in event driven scenarios is an important issue. Such a congestion can occur not only in data communication network but also in other networks where items (cars, packets, people etc.) can sporadically appear and disappear as a consequence of an event. Using everyday life use-case scenario, we show that our work has significant impact, and our approach can be applied to alleviate real world problems arising from congestion.

Various cities often host festivals and games in which large crowds of people participate. Imagine a city hosting a festival that attracts large audience. At the end of the festival, the festival area empties and participants try to leave through their nearest exits and via shortest paths. There are limited number of exits. Moreover, some exits might be closer to car parks or bus stations which, if not controlled, will draw more crowd. Therefore, such exits are more prone to congestion, Hence, any mismanagement of these exits can easily cause stampede. However, all exits can be monitored via video cameras and their ingress and egress ratios can easily be computed. The movement of the people will provide an estimate of how fast people are passing through exit. Thus, our mechanism can predict congestion by dynamically computing the total passage time on each exit route. Using this information, electronic displays strategically placed at cross-paths, can guide dynamically groups of people to an exit that currently offers the fastest passage time. Displays will periodically change their recommended route as the exits change their passage time according to the numbers of people passing through them, thus avoiding stampedes. Such event driven routing guidance and management of physical infrastructure can save human lives. Recently, a stampede in a music festival in Germany killed a number of people, leaving several injured. The stampede occurred when the crowd departing festival followed the shortest path and the exit was too narrow to cope with huge traffic [6]. An early divergence of people to different exits and effective monitoring could have saved precious lives. Unfortunately, such disasters are quite frequent, as evidenced by [7] that reviews major disasters in mass gatherings over past decades caused by stampedes and mismanagement of evacuation routes.

The scenarios described above show how bursts of flow entities (packets in communication networks, people in tunnels or cars on the roads) create congestion and cause delays in transmission. In the above case scenarios the application of our proposed mechanism enables us to efficiently route the prioritized flow entities and efficiently disperse them (i.e., 
packets, people or cars) by dynamically routing them via multiple paths and leverage all possible paths to reduce and possibly avoid congestion.

\section{RELATED WORK}

Congestion control is well studied area of computer networks; however, congestion control in event driven scenarios is still an open challenge. Various routing protocols have been devised to efficiently route traffic to destination. Researchers have studied feedback loop based protocols and buffer control protocols to alleviate congestion. The congestion control techniques that use feedback loops to adjust data generation rate are not applicable to event driven scenarios because the event may disappear by the time rate is adjusted. Moreover, in scenarios where network is partially damaged, the feedback loop generated control traffic in the network may lead to lower throughput of the network. It is important to note that multipath routing for congestion control has been studied in the past but most of the proposed approaches do not assume event driven traffic. Moreover, to the best of our knowledge none of the proposed approaches dynamically route prioritized traffic to multiple paths based on auction winning prices. Our route assignment approach is different from pure link delay based route assignment. We take into account the nature of traffic on the link, which is why a link may cause delay to a low priority traffic, but can be faster for high priority traffic. So each link has different delay for applications of different priorities.

A distributed auction based mechanism to resolve congestion in distributed manner is proposed in [8]. In this approach every candidate packet bids its predicted utility loss which it will incur, if it is delayed for a transmission slot. The packet of application with highest bid is chosen for transmission at each time slot. However, the proposed approach makes a local decision to assign a transmission slot and the routing decision is not the focus of the work. Moreover, the authors do not address congestion control in case of network failures. In [9], the authors propose a receiver-assisted congestion control mechanism using Transmission Control Protocol (TCP). In their approach the receiver notifies the sender about the estimated rate of transmission to avoid congestion. Such a rate control mechanism or similar TCP based approaches are not feasible for event driven traffic as explained above.

In buffer management technique as proposed in [10], nodes maintain buffer states which are piggybacked to their neighbors. The buffer state of a node tells its neighbors if they should send packet to it. In such a way, packet transmission rate in whole network is adjusted. The authors have also incorporated multipath routing in their mechanism in which the priorities are statically assigned based on link conditions. Static assignment of links based on priority may overload links with less delay. In [11], the authors present a congestion control technique for prioritized traffic that uses piggybacking of congestion notification embedded in packet's header. Each node is assigned certain priority index. Once congestion is detected, bandwidth proportional to the priority index of the node is allocated to the traffic generated by that node. This approach also uses rate control mechanism to avoid congestion and may suffer from issues mentioned earlier for rate control approaches. The disadvantage of such assignment is that a low priority node may receive packets that are of high value that will incur large delays due to node's low priority. A prioritized multipath approach is suggested in [12] to minimize end to end delay. This approach also statically assigns paths based on priorities so it suffers from shortcomings as mentioned earlier.

Route recovery protocols to alleviate failures have also been studied in the past. In [2], authors propose a protocol which detects the network failures by packets losses from the sender nodes. Once the failure is detected and failed nodes are identified, the routing interval is increased to notify the neighboring nodes of the failure. After that, alternate paths to the child nodes of the failed parent nodes are discovered which are then used for further communication. In such an approach, alternate routes are discovered only in case of failures. A path with congestion is still considered as usable and the traffic is not rerouted in such cases. In our approach, we not only reroute packets in case of failures, but also in case of congestion. Moreover, routing packets from multiple applications of various priorities, which we are focusing on, is not addressed in their approach. Other multipath routing approaches based on link weight and dynamic link weight proposed in [13], [14] are highly dependent on the quality of weight assigned to the links.

Research community working on building evacuation and road traffic congestion management in event driven scenarios is facing similar challenges as computer network researchers. We have discussed in Section II, relationship between guiding people through safe exits in buildings and routing in communication networks. In [4], researchers have devised navigation system to enable efficient evacuation of buildings in case of emergency. In their approach two kinds of nodes (sensing and decision nodes) facilitate the evacuation of habitants from a building. Based on information collected from sensing nodes, decisions nodes compute effective shortest path to guide people out of the building.

\section{Dynamic PRice Based Routing}

In order to maximize the network throughput and decrease transmission delay for all the applications, we propose the following mechanism. Whenever a router has a packet of certain application to deliver to the next hop towards destination, it looks at all of its neighbors which can lead a route to the destination without looping. The router then uses the price for each path to estimate the total delay that the packet will encounter if this path is selected. Then, the router sends the packet on the path on which the delay is the smallest. An auction is held and path with lowest price is selected and packet is forwarded to the neighbor leading that path to destination.

The path prices are proportional to the predicted time delay for a packet of a unit priority taking the path. This time delay is a function of the path congestion. The path price 
can be translated into waiting time of a packet with a given priority, but the mapping depends on routing and transmission protocols and technical details of the network used. It is important to notice that path price computation is based only on information obtained from the immediate neighbors of the router. Each router calculates prices for route originating from each of its neighbors but not all nodes in the network. The same processing repeats on every hop and a minimal cost route is selected on hop by hop bases which leads to overall minimal delay path.

A path may have different delays for applications with different priorities. A path that imposes long delay for low priority application may impose short delay for a high priority application which eventually leads to segregation of different types of traffic in the network. Moreover, as the state of the network changes over time and congestion appears and disappears at different points of the network, the path prices follow and change dynamically. This is why in our proposed mechanism paths are not statically allocated to different traffic categories and traffic pertaining to an application does not follow a single path but changes its path according to the network conditions. As our protocol routes each packet to node which leads to lowest cost route at each step, there is chance that the packet gets into a routing loop, in which it keeps oscillating between some nodes and never gets to destination. In order to avoid such loops, each node checks the number of hops to destination from its neighboring nodes. The candidates for next hop are restricted to those neighboring nodes which have fewer hops to destination than the current node. Thus the packet always moves forward towards destination (downstream movement) strictly decreasing its number of hops to destination and avoids routing loops. It is important to note that the packet may choose longer path over shorter available path but in both cases the next hop has to have fewer hops to destination than the current one to avoid routing loop. In our protocol, the main goal is to find the path with minimal cost, therefore each node has a routing table which contains routes to destination via its neighbors. This table can be easily populated only once using Dijkstra's algorithm or any other path finding algorithm. With such a routing table, every node knows its distance in hops to destination.

Figure 1(a) shows a simple network and three applications with priorities represented by number 1,5 and 30 where higher number represents higher priority. Packets generated for three applications are routed to the same destination. There are three routes of different lengths from source to destination. Here we assume the following simple routing protocol. At each router, if there are packets competing for transmission slot, they bid for the right to use this slot. The highest bid wins and becomes the price for the paths passing through this node. The initial bid of each packet is its priority. Each packet losing the auctions increases its bid for the next auction by adding to the old bid its priority. For this simplistic scenario, the bids are shown in the figure 1. 'a3' has the highest bid, it goes through the shortest path. Slightly longer paths are taken by applications 'a1' and 'a2'. Node ' $\mathrm{J}$ ' which leads to shortest

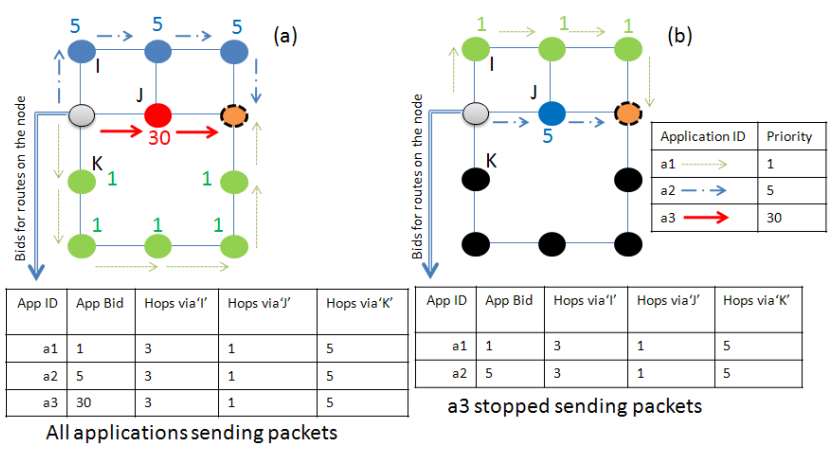

Fig. 1. Route selection based on path price

path to the destination will be avoided by applications 'al' and ' $a 2$ ', because packets from ' $a 3$ ' which are high priority will most of the time win the transmission slot on node ' $\mathrm{J}$ ' and other applications would need to wait till through the auction losses they accumulate a bid with which they can win auction on node ' $\mathrm{J}$ '. This is an unnecessary contention as other routes to destination are available. Therefore, our mechanism wisely sends other applications through alternative routes as shown in figure 1 . If the network state changes and for certain period there are no more packets of application ' $a 3$ ' flowing through node ' $J$ ', routing changes. The winning bid on node ' $\mathrm{J}$ ' changes as the router automatically reconfigures the routes. As seen in figure 1(b), packets from 'a2' which have the second highest priority go through the shortest path and packets from 'a1' go through the route previously used by 'a2'. Thus, the routing nodes dynamically reconfigure paths based on the network conditions indicated by prices on neighboring nodes. Consequently, the routes are not underutilized like in the case of statically assigned routes.

\section{Dynamic Path Selection with predicted Quality of Information loss}

Suppose there are several object tracking applications hosted on a WSN, monitoring different objects of different priorities. Every application has a utility function which represents its Quality of Information (QoI) that decreases proportionally to the product of the accumulated communication delay and priority of the applications. Every time unit, the router transmits one packet. We incorporate auction mechanism described in [8] for selecting winner of the transmission slot. Assuming TDMA as medium sharing protocol, application $i$ bids its QoI loss represented by the following utility function.

$$
b_{i d}=\Delta t p_{i} t_{c}
$$

where $p_{i}$ is the priority of the application $i, t_{c}$ is the TDMA cycle time and $\Delta t$ represents the difference between packet generation time of current bidding packet and the packet that the router transmitted previously for application $i$. Please note that the $\Delta t$ increases as consequence of losing auctions and therefore the bid also increases for application $i$. Whenever there is congestion on the routing node, the auction winning bid increases and applications with higher bids go through 
while applications with lower bids have to wait and compete in upcoming auctions. We also replace old packets with new packets in the queue as transmitting fresher packets will prevent more QoI losses compared to sending older packets with or without new ones.

Once the winner of transmission slot is selected, it needs to be transmitted to one of the downstream neighbors. As discussed above, as congestion arises on a downstream node, the winning bid increases on that node. In this scenario, the auction winning bid acts as the price of a path originating in this node because the packet has to wait on the node and win auction before it can take path originating from this node. Using this price, we can estimate the transmission delay on routes originating from this node. Use of TDMA protocol with its transmission cycles makes the path delays well predictable, but other techniques for delay prediction can be used for protocols other than TDMA. The path price divided by the packet priority in this case represents the number of TDMA cycles that the packet needs to wait until it gets a transmission slot for the next hop.

To summarize, when a packet attempts to make the next hop, it may need to compete with packets from other applications for transmission slot on that node. This competition can be based on any mechanism, here we assume auctions as described above to estimate waiting time on the node. In order to win auction on this new node, the packet has to wait and accumulate QoI losses proportional to packet's application priority before it can win the auction and get the transmission slot. This is important to note that, if the next-hop is congested and high priority packets are bidding in auction, then a low priority packet will have to wait longer and suffer bigger delays before it can make up a winning bid. Therefore, selecting next hop for a packet is crucial and can lead to longer delays if low priority packets join auction with high priority packets. We have noticed that this problem arises when all the packets try to go through the shortest path and end up in high QoI loss. Our model intelligently tackles this situation by separating high priority packets from low priority packets. In our model each packet compares its priority with the winning bids on the candidate next-hop; from this comparison, it can calculate how long it would need to wait until it wins auction for the transmission slot. Moreover, it also takes into account how many more hops it needs to travel from the next hop onwards, before it can reach the destination. This gives us predicted delay on the candidate next-hop and thus on the path to destination originating from that hop. The path prices are reflection of such delays. The following equation represents path price denoted as $P_{i}^{k}$ on neighbor node $k$ for application $i$.

$$
\begin{gathered}
P_{i}^{k}=H^{k} \times\left(\frac{H b i d^{k}}{M b i d_{i}^{k}}+\frac{1}{2}\right) \\
\text { Next hop }=k \text { with } \min P_{i}^{k}
\end{gathered}
$$

where $H^{k}$ is the number of hops to destination from neighbor node $k, H b i d^{k}$ is the price i.e. the highest or winning bid on node $k, M b i d_{i}^{k}$ is the bid of the application $i$ (hence the bid of the packet seeking the next hop) on node $k$. There is an underlaying assumption that a node overhears its neighbor's highest bid at the time this neighbor sends forwarded packet for application $i$ which enables calculation of $M b i d_{i}^{k}$ on node $k$. These parameters can be embedded in packet's header in forwarding procedure. This way, whenever one node forwards packet to another, the neighboring nodes (which may not be the destination nodes for this packet) can also read these parameters from header. This is a reasonable procedure because all the neighboring nodes need to read header for destination address, to find out if the packet is destined to them. Therefore reading two extra parameters will consume little energy and processing power. There are efficient overhearing techniques already proposed in the literature which can be incorporated for overhearing.

Our QoI function is linear in priority, therefore in our scenario, the ratio between the application $i$ 's bid and the winning bid on the neighboring node acts as good predictor of waiting time for transmission slot. Consequently, we can predict the waiting time on next node using $H^{k} \times\left(\frac{H b i d^{k}}{M b i d^{k}}\right)$, because depending upon what is the highest wining price on the next node, the packet will need to wait accordingly. The average future delay without any congestion is $H^{k} / 2$. In other words, the price of auction on the next node translate to the waiting time on that node. The same mechanism can be easily generalized for other QoI functions based on the priority of the application, so the prices on the next node can be estimated. Another important point to notice in equation (2) is that, as more and more packets follow certain path, the winning price on that node will increase and the prices will reflect the predicted delay on the node. Eventually, routers will start diverting low priority applications packets to other nodes where the auction prices are lower. In such a way, the dynamic prices on nodes will automatically redirect the traffic as congestion increase on the nodes.

In our proposed mechanism the starvation of the packets is avoided by using aging effect. For each application, the bid is defined by the priority as well as the waiting time. So, as the packet of an application loses auctions, its loss of QoI increases, increasing the bid of the application. After losing some auctions, the application bid eventually rises enough (due to aging) that it will beat other (even high priority) applications in the auction and the corresponding packet is transmitted.

\section{Performance Evaluation}

We implemented the price based routing (PBR) protocol in NS2 [15] and simulated object tracking scenario. We use TDMA as MAC level protocol and TwoRayGround as radio propagation model. We simulate scenario in which objects such as cars moving on various roads in a city are tracked by wireless sensors e.g., video cameras stationed at different locations in the city. The cars move in and out of the coverage areas of different cameras. The data is sent over the wireless network to the control center where it is monitored by security agencies of the city. Depending upon the driving pattern of the 
cars the security chief assigns priorities to suspicious vehicles to keep close eye on them. Whenever the target vehicles enter the sensing range of the camera, the camera starts sending large number of packets to the control center to provide full information about the location of the vehicles. When the target vehicles leave the sensing range of the camera, the camera switches back to normal mode and sends low fidelity data to control center. Similarly, every camera produces large number of packets whenever target vehicles enter their sensing range. Such bursts of data create congestion on downstream nodes in the sensor network causing packet loss and increased delay in packet delivery.

We modified the DSDV protocol in NS2 to implement our proposed protocol represented by PBR in the results and compared it with the performance of native DSDV protocol in NS2 distribution. Simulated sensor network consists of 44 nodes with one destination and multiple mobile objects (represented by NS2 nodes without routing and sensing capabilities) moving in the field to mimic real life objects (e.g. cars). We experimented with number of objects varying between 2 and 4 , where each object is served by a different object tracking application. The sensing nodes were fixed for all experiments whereas the mobile objects moved around with certain mobility patterns while being tracked by fixed sensing nodes. For our experiments we simulated three different mobility models: "Random Waypoint Model (RWM)", "Pursue Mobility Model (PMM)" [16] and "Manhattan Grid Model (MGM)". For every mobility model we ran simulations for 500 seconds with various priorities ${ }^{2}$. The priority of an application increases with the number that represents it and is set by the consumer of the information. The results presented in this section are averaged over 7 different runs.

\section{A. Fully Functional Network}

In order to show the efficiency of our routing protocol in a fully functional network, we simulated network without any failed nodes or broken paths. We measured the sum of QoI losses for all the applications for both DSDV protocol and our dynamic price based routing protocol. Figure 2 presents the percentage decrease in QoI loss when our proposed mechanism is used as compared to DSDV routing protocol. As the figure shows, our approach decreases the utility loss by one third, in some cases. We observed that our method saves QoI losses. The percentage gain over DSDV in our results was calculated using equation 4 .

$$
\text { Gain }=100 \times \frac{\text { QoILoss }_{D S D V}-\text { QoILoss }_{P B R}}{\text { QoILoss }_{P B R}}
$$

where $\operatorname{QoILoss}_{P B R}$ is the QoI loss in case of our protocol and QoILoss $D S D V$ is the QoI loss observed in case of DSDV.

Figure 5 shows the decrease of the QoI losses for applications under our PBR protocol as compared to DSDV. In the

\footnotetext{
${ }^{2}$ Priorities: $\quad\{(5,10), \quad(9,36), \quad(8,64), \quad(16,64), \quad(27,216), \quad(4,16)$, $(10,20)\},\{(5,10,15), \quad(9,36,81), \quad(8,64,216), \quad(16,64,144), \quad(27,216,729)$, $(4,16,36), \quad(10,20,30)\},\{(5,10,15,20), \quad(9,36,81,144), \quad(8,64,216,512)$, $(16,64,144,256),(27,216,729,1728),(4,16,36,64),(10,20,30,40)\}$
}

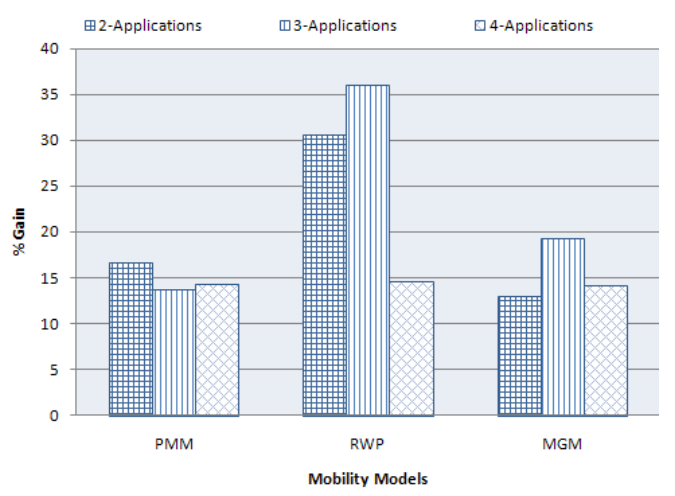

Fig. 2. Percentage decrease in QoI loss in price based routing compared to DSDV under different mobility models and multiple applications

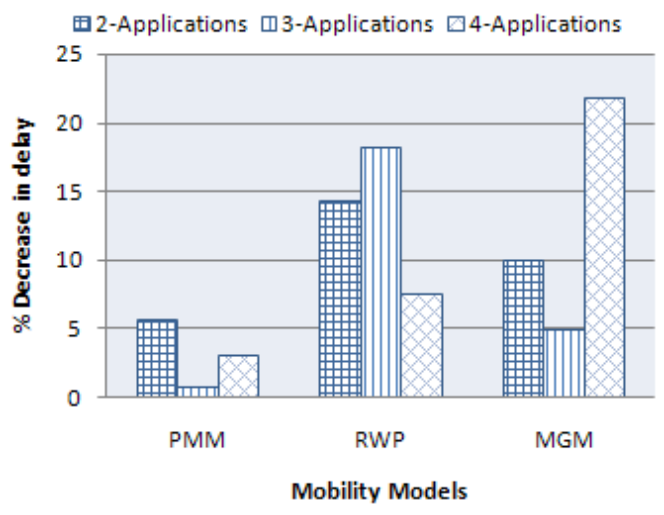

Fig. 3. Percentage decrease in packet delivery delay in price based routing compared to DSDV under different mobility models and multiple applications

interest of space, measurements for only one priority set and MGM model are presented. Please note that even with DSDV, we use auction mechanism for slot winner selection. In case of DSDV runs shown in all the experiments, when winner of transmission slot is selected using auction mechanism [8], the packet is routed using DSDV protocol as opposed to our proposed routing mechanism.

Figure 3 shows decrease in average packet delivery delay. The figure shows that our intuition of dynamically routing traffic with different priorities on different paths actually decreases delay for all the applications in different scenarios. This is because when the mechanism notices that the auction winning prices on certain route are increasing, it diverts traffic from that route to alternative routes to prevent the increase in congestion on that route. This leads to increased packet delivery. As shown in the figure 4, our algorithm delivers $15 \%$ more packets than DSDV protocol can.

\section{B. Partially Damaged Network}

Our proposed mechanism is resilient to network changes by dynamically adjusting paths in case some portion of the network fails and some nodes are not operational. To show that capability, we simulated a scenario in which the network partially fails during normal operation because of some emergency or component failure. We evaluated the performance of 


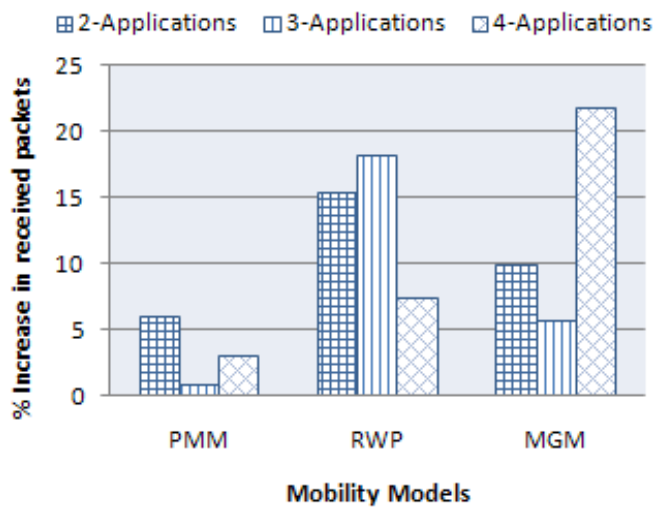

Fig. 4. Percentage increase in packet received in price based routing compared to DSDV under different mobility models and multiple applications

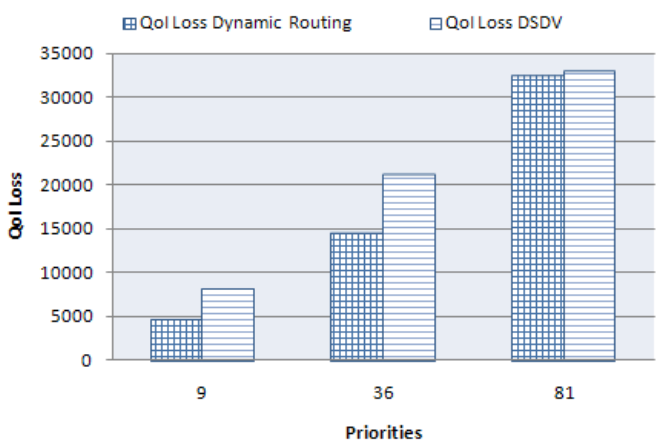

Fig. 5. QoI losses for three applications under dynamic price based routing and DSDV protocol (note that that total loss of QoI in this scenario is the sum of losses for all three applications, so clearly lower priority application gain the most from our mechanism).

our mechanism in cases where $5 \%, 10 \%$ and $30 \%$ of the network is damaged and nonfunctional to test the responsiveness and resilience of our protocol with increasing network failures. In this section, we present simulation results showing that our algorithm performs well in a partially damaged network. Figure 6 shows performance comparison for a network in which $5 \%$ of the nodes fail in middle of simulation. We can see in the figure 7 that our protocol performs better than DSDV and even with $5 \%$ of damage, the performance is comparable to fully functional network. With our approach we can increase the number of delivered packets and decrease delay.

As we increase damage to the network, the performance of our protocol decreases as expected. As more and more sensing nodes fail, we have fewer tracking packets generated as well as fewer routing nodes. Therefore throughput of the network decreases, but by leveraging the available network, the effect of the damage can be decreased. With $10 \%$ damage to the network, our protocol still reduces QoI losses and decreases the delay by around $15 \%$. Please note, that the gain, decrease in delay and increase in packet delivery, also depends on the mobility model assumed by the moving objects. For example, we have lower gains in PMM model because objects in pursue model follow each other, thus they move in a group. All the objects in the group are usually detected by the same

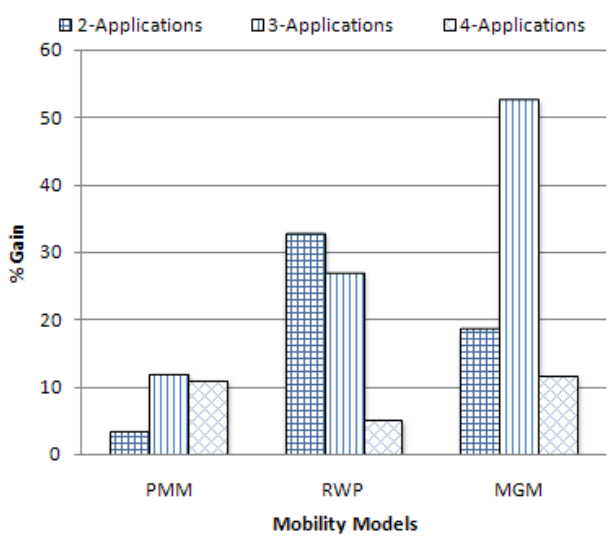

Fig. 6. Percentage gain in dynamic priced based routing compared to DSDV under different mobility models and multiple applications in 5\% damaged network

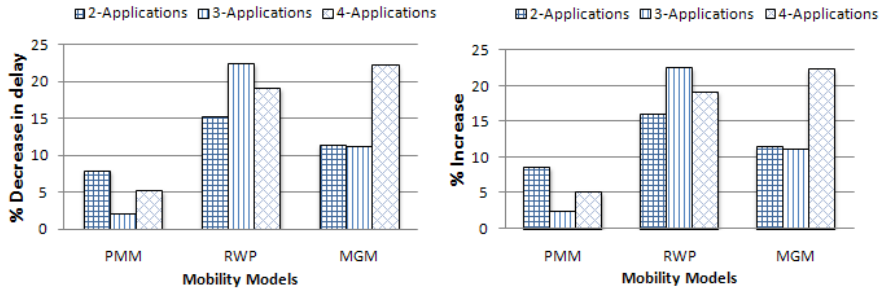

Fig. 7. Percentage increase in packet delivery and decrease in delay, in dynamic price based routing compared to DSDV under different mobility models and multiple applications in 5\% damaged network

group of sensing nodes, therefore the sum of QoI loss is smaller when the network is fully operational. When the network is damaged and all the objects move to damaged part of the network, we have much lesser information about all the objects, thus incurring high QoI losses, which may be unavoidable. When objects move in an uncoordinated way, like in RWP and MGM models, we may have one object in damaged part of the network but others detected by the operational part of the network, which reduces the overall QoI loss. This enables our routing algorithm to disperse the traffic through different routes. Please note that in $30 \%$ highly damaged network, we can still reduced packet delivery delay and improve packet delivery significantly in RWP and MGM models but the performance with PMM model is lower, as explained earlier.

\section{CONCLUSION}

In this paper, we have investigated a novel dynamic price based routing protocol for prioritized traffic in both communication networks and physical infrastructure networks. We show that routing packets based on path prices improves routing decisions and helps to reduce congestion in the network, by dynamically establishing partially or fully disjoint paths for multiple applications. The packets with lower priority may incur higher delays if routed on shorter paths that are congested. Therefore, our mechanism enables low priority packets to go through alternate paths which may be longer, 


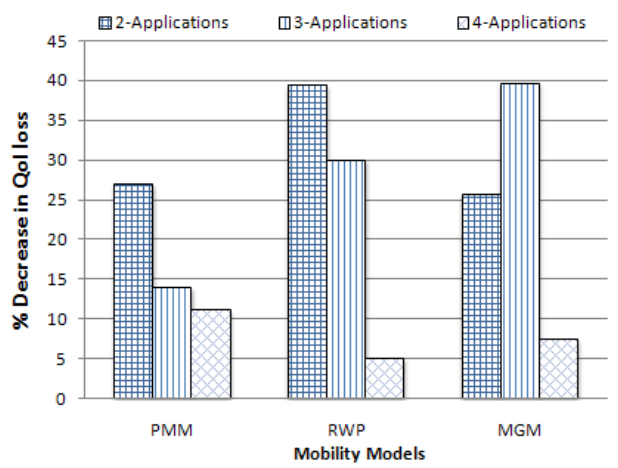

Fig. 8. Percentage gain over DSDV under different mobility models and multiple applications in $10 \%$ damaged network
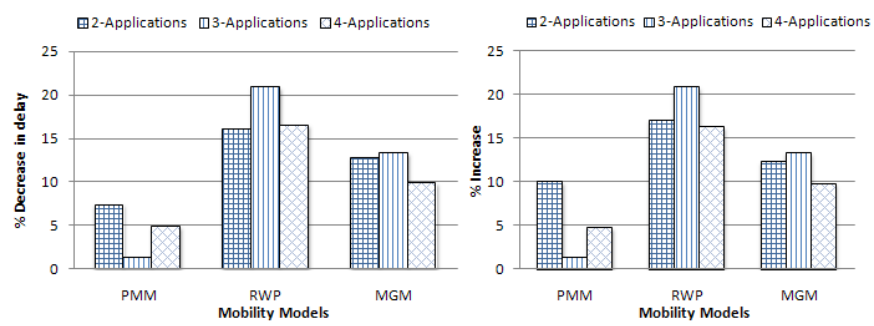

Fig. 9. Percentage increase in packet delivery and decrease in delay, in dynamic price based routing compared to DSDV under different mobility models and multiple applications in $10 \%$ damaged network

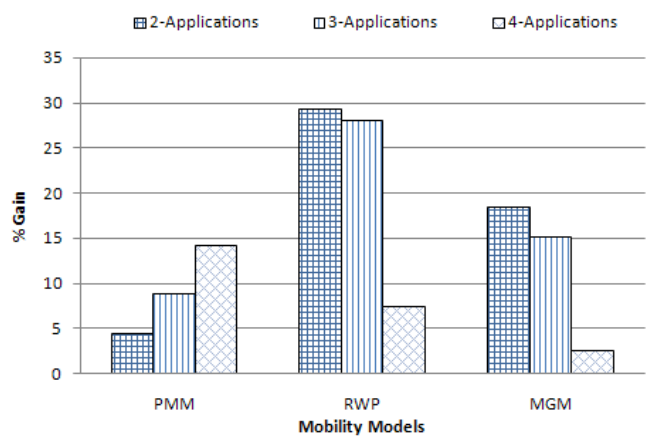

Fig. 10. Percentage gain over DSDV under different mobility models and multiple applications in 30\% damaged network
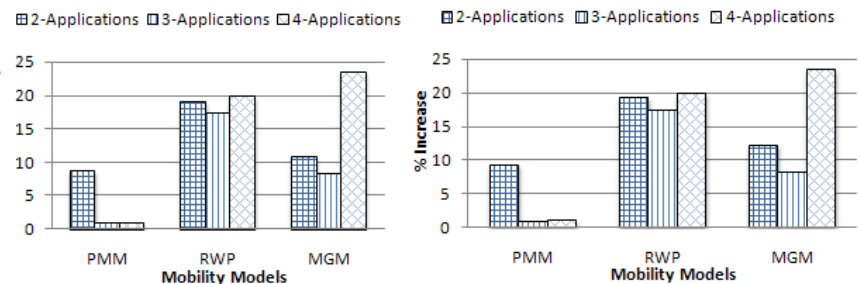

Fig. 11. Percentage increase in packet delivery and decrease in delay in dynamic price based routing compared to DSDV under different mobility models and multiple applications in $30 \%$ damaged network

but due to lighter traffic impose shorter delays. We also show that our approach dynamically segregates multi-priority traffic in network making it robust against partial network failures. As our mechanism is not tightly coupled with data communication network protocols, it is equally applicable to physical infrastructure networks for routing spontaneous bursts of people and vehicles from one point to another.

\section{ACKNOWLEDGMENT}

Research was sponsored by the Army Research Laboratory and was accomplished under Cooperative Agreement Number W911NF-06-3-0001. The views and conclusions contained in this document are those of the authors and should not be interpreted as representing the official policies, either expressed or implied, of the Army Research Laboratory, the U.S. Government, the UK Ministry of Defense, or the UK Government. The US and UK Governments are authorized to reproduce and distribute reprints for Government purposes notwithstanding any copyright notation here on.

\section{REFERENCES}

[1] E. Gelenbe, G. Gorbil, and F. Wu, "Emergency cyber-physical-human systems," in Computer Communications and Networks (ICCCN), 2012 21st International Conference on. IEEE, 2012, pp. 1-7.

[2] J. Lee and I. Jung, "Speedy routing recovery protocol for large failure tolerance in wireless sensor networks," Sensors, vol. 10, no. 4, pp. 3389$3410,2010$.

[3] D. Johnson and D. Maltz, "Dynamic source routing in ad hoc wireless networks," Mobile computing, pp. 153-181, 1996.

[4] G. Gorbil, A. Filippoupolitis, and E. Gelenbe, "Intelligent navigation systems for building evacuation," in Computer and Information Sciences II: 26th International Symposium on Computer and Information Sciences. Springer Verlag, 2011, p. 339.

[5] M. Li, Y. Liu, J. Wang, and Z. Yang, "Sensor network navigation without locations," in INFOCOM 2009, IEEE. IEEE, 2009, pp. 2419-2427.

[6] 2010. [Online]. Available: http://www.guardian.co.uk/world/2010/jul/24 /love-parade-festival-tunnel-stampede [JAN,16,2013]

[7] L. Soomaroo and V. Murray, "Disasters at mass gatherings: Lessons from history," PLoS currents, vol. 4, 2012.

[8] L. Chen, Z. Wang, B. Szymanski, J. Branch, D. Verma, R. Damarla, and J. Ibbotson, "Dynamic service execution in sensor networks," The Computer Journal, vol. 53, no. 5, p. 513, 2010.

[9] K. Shi, Y. Shu, O. Yang, and J. Luo, "Receiver-assisted congestion control to achieve high throughput in lossy wireless networks," Nuclear Science, IEEE Transactions on, vol. 57, no. 2, pp. 491-496, 2010.

[10] S. Chen and N. Yang, "Congestion avoidance based on lightweight buffer management in sensor networks," Parallel and Distributed Systems, IEEE Transactions on, vol. 17, no. 9, pp. 934-946, 2006.

[11] C. Wang, B. Li, K. Sohraby, M. Daneshmand, and Y. Hu, "Upstream congestion control in wireless sensor networks through cross-layer optimization," Selected Areas in Communications, IEEE Journal on, vol. 25 , no. 4 , pp. 786-795, 2007.

[12] X. Huang and Y. Fang, "End-to-end delay differentiation by prioritized multipath routing in wireless sensor networks," in Military Communications Conference, 2005. MILCOM 2005. IEEE. IEEE, 2005, pp. $1277-1283$.

[13] B. Fortz and M. Thorup, "Optimizing ospf/is-is weights in a changing world," Selected Areas in Communications, IEEE Journal on, vol. 20, no. 4, pp. 756-767, 2002.

[14] S. Liu, X. Zhang, J. Yu, Y. Liu, and X. Chen, "A dynamic traffic distribution strategy for multipath routing," in Information, Communications and Signal Processing, 2009. ICICS 2009. 7th International Conference on. IEEE, 2009, pp. 1-5.

[15] [Online]. Available: http://www.isi.edu/nsnam/ns/ [JAN,16,2013]

[16] T. Camp, J. Boleng, and V. Davies, "A survey of mobility models for ad-hoc network research," Wireless Communications and Mobile Computing, vol. 2, no. 5, pp. 483-502, 2002. 